\title{
ABLAÇÃO SELETIVA DE FILME FINO DE TIN DEPOSITADO SOBRE UM SUBSTRATO DE WC*
}

Eduardo Spinelli Oliveira1 Ricardo Elgul Samad²

Nilson Dias Vieira Junior ${ }^{3}$ Wagner de Rossi ${ }^{4}$

\begin{abstract}
Resumo
Devido à possibilidade de se remover quantidades muito pequenas de material de cada vez, a ablação seletiva por laser tem se mostrado um método muito eficaz para remoção de finas camadas de materiais depositados sobre um substrato. Neste trabalho, um laser de femtossegundo foi utilizado para remoção de um filme fino de nitreto de titânio (TiN) depositado sobre um substrato de carboneto de tungstênio (WC), configuração comum em pastilhas de usinagem mecânica, objetivando fornecer um método eficiente para tal processo. Os limiares de ablação do filme e do substrato foram determinados, evidenciando a problemática do trabalho, remover um filme com limiar maior que o substrato. Um sistema de controle de espectrometria de emissão atômica induzida por laser (LIBS) foi testada, com a intenção de controlar em tempo real, a espécie do material ablacionado, mostrando-se muito eficiente. Os parâmetros do laser bem como da varredura de usinagem ainda estão sendo testados para definir a viabilidade do processo.
\end{abstract}

Palavras-chave: Laser de femtosegundo; Ablação seletiva; Microusinagem; Filmes finos.

\section{SELECTIVE ABLATION OF A THIN TIN FILM DEPOSITED ON A TUNGSTEN SUBSTRATE}

\section{Abstract}

Due to the ability to remove very small amounts of material at a time, the selective laser ablation has proven a very effective method for removing thin layers of material deposited on a substrate. In this work, a laser femtosecond was used to remove a thin film of titanium nitride (TiN) deposited on a tungsten carbide substrate (WC), common configuration for mechanical machining chips, aiming to provide an efficient method for this process. The film and substrate ablation threshold were determined, highlighting the problems of work, remove a film with threshold higher than the substrate. An laser induced atomic emission spectrometer control system (LIBS) has been tested with the intention of controlling in real time the species of material ablationed, proved to be very efficient. The laser and machining parameters are still being tested to determine the availability of the process.

Keywords: Femtosecond laser; Selective ablation; Micromachining; Thin films.

1 Licenciatura em Matemática, Licenciado, Aluno, Centro de Lasers e Aplicações, Instituto de Pesquisas Energéticas e Nucleares, São Paulo, SP, Brasil.

2 Bacharelado em Física, Doutor, Pesquisador, Centro de Lasers e Aplicações, Instituto de Pesquisas Energéticas e Nucleares, São Paulo, SP, Brasil.

3 Bacharelado em Física, Doutor, Pesquisador, Centro de Lasers e Aplicações, Instituto de Pesquisas Energéticas e Nucleares, São Paulo, SP, Brasil.

4 Bacharelado em Física, Doutor, Pesquisador, Centro de Lasers e Aplicações, Instituto de Pesquisas Energéticas e Nucleares, São Paulo, SP, Brasil. 


\section{INTRODUÇÃO}

Filme fino é um termo utilizado para designar camadas de materiais que vão desde frações de nanômetros até vários micrômetros de espessura, embora ainda haja uma discussão quanto ao limite dessa espessura [1]. A tecnologia de filmes finos vem sendo cada vez mais utilizada, principalmente em tecnologias de armazenamento de dados (óptico e magnético), telecomunicações, displays, células de combustível, células fotovoltaicas, sensores, atuadores, além de recobrimentos com diversas funções (ópticas, decorativas, proteção ambiental, proteção ao desgaste mecânico, como barreiras de difusão), dentre outros [2].

Os principais processos de fabricação de filmes finos são a modificação da superfície do substrato por altas temperaturas (tratamento térmico) e a deposição de material vaporizado (sputering). Muitas vezes é necessário controlar, impedir o crescimento do filme ou até mesmo eliminar a camada em determinadas regiões da superfície, como por exemplo, para fabricação de células fotovoltaicas. Um dos métodos utilizados para remoção de filmes depositados em áreas específicas é a ablação seletiva por laser, que, com 0 avanço das técnicas de controle e equipamentos cada vez mais precisos, vem ganhando espaço nos processos industriais de fabricação [3].

A ablação seletiva por laser, consiste na remoção de camadas individuais de material a partir de um substrato multicamadas, preferencialmente, sem danificar o material que não se deseja eliminar. A diferença entre as propriedades físicas do filme e do substrato determinam a possibilidade, a precisão e a complexidade do processo. Se o material a ser removido absorve o comprimento de onda do laser, e apresenta um limiar de ablação mais baixo que o do substrato, então o processo é relativamente simples e não há necessidade de um controle de realimentação. $A$ fluência do pulso é ajustada para estar um pouco acima do limiar de ablação do filme e abaixo do limiar do substrato, assim, um excesso de pulsos sobre a superfície do substrato não irá afetá-lo. Por outro lado, se o limiar de dano do recobrimento é próximo ou menor que o do substrato, então um controle realimentado pelo processo deve existir para evitar dano excessivo ao substrato. Isto pode ser feito através da medida, em tempo real, do espectro do plasma emitido pela interação do filme com o pulso laser focalizado, utilizando a técnica de espectrometria de emissão atômica induzida por laser (LIBS - Laser-induced Breakdown Spectroscopy) [4], observando a evolução das emissões características do filme e do substrato. Esta análise permite determinar com precisão quando o material do revestimento está acabando e quando o material de substrato começa a ser ablacionado pelo laser.

Para remoção de filmes de nitreto de titânio (TiN) depositados sobre um substrato de carboneto de tungstênio (WC), combinação comum em pastilhas de usinagem, a ablação realizada por lasers de pulsos com largura temporal de nanossegundos não seria aconselhável, pois a ablação se dá por meio de processos térmicos, onde uma grande parte do material é ejetado na forma de vapor, e outra parte é fundida e ressolidificada, permanecendo nos arredores da superfície ablacionada. Além deste inconveniente, uma zona afetada pelo calor (ZAC) também é formada nos arredores da região irradiada pelo laser. A espessura desta região depende principalmente da energia utilizada, da largura temporal do pulso laser, e das propriedades térmicas do metal processado. A formação destas camadas, de regiões ressolidificadas e de ZAC, com formação de fases cristalinas diferentes daquelas do metal original, levam 
a uma modificação física e metalúrgica, que podem não mais ser adequadas à sua utilização como ferramenta de corte.

Para realizar a ablação seletiva sem provocar estes efeitos negativos, uma possibilidade é a utilização de lasers de pulsos ultracurtos, com larguras temporais da ordem de poucas dezenas ou centenas de femtossegundos ( $\left.1 \mathrm{fs}=10^{-15} \mathrm{~s}\right)$. Como a duração temporal destes pulsos é menor que o tempo de interação elétron-fonon, grande parte do processo de ablação ocorre antes que haja transferência significativa de calor para a rede cristalina, e o material é ejetado por repulsão coulombiana, sem que haja acúmulo significativo de calor na região de interação [5, 6].

Para determinar o limiar de ablação de um material por lasers de pulsos ultracurtos, ou seja, determinar a quantidade mínima de energia capaz de causar algum dano ao material, utilizou-se a técnica D-Scan, desenvolvida no laboratório de lasers de altíssima intensidade do CLA-IPEN [7], que consiste na varredura da amostra nos sentidos transversal e longitudinal, simultaneamente, conforme mostra a Figura 1(a), indo de um ponto anterior à cintura do laser até um ponto posterior. Esse processo permite, em determinados níveis de intensidade do laser, a gravação de um perfil simétrico na amostra, conforme mostra a Figura 1(b). Através das dimensões do perfil gravado a dos parâmetros utilizados na varredura, é possível determinar os limiares de ablação para os regimes de baixa e alta fluência com boa precisão $[7,8]$.

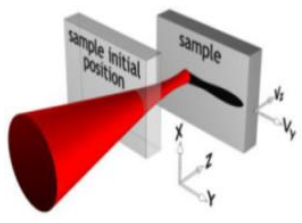

(a)

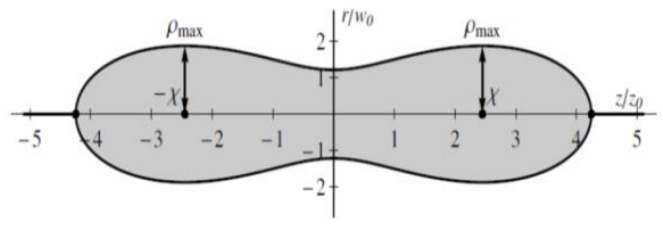

(b)

Figura 1. a) Esquema da varredura diagonal da amostra; b) perfil gravado na amostra. [5]

A simples utilização de pulsos ultracurtos, contudo, não garante a interação não térmica do processo, e somente a utilização de parâmetros adequados do pulso laser podem levar a um processo com mínimo acúmulo de calor. Assim, há a necessidade de se encontrar os parâmetros de processo que levem a um acúmulo mínimo de calor na região de interação com o pulso laser, e, ao mesmo tempo, garantam a remoção completa do filme depositado sobre a pastilha de usinagem.

\section{MATERIAIS E MÉTODOS}

\subsection{Laser de pulsos ultra-curtos}

Os experimentos foram realizados no laboratório de lasers de altíssima intensidade e no laboratório de aplicações industriais, ambos localizados no Centro de Lasers e Aplicações (CLA) do Instituto de Pesquisas Energéticas e Nucleares (IPEN).

O sistema consiste de um laser de Ti:Safira de femtossegundo, amplificado por varredura de frequência (Femtopower Compact Pro CE-Phase HP/HR da marca Femtolasers) que gera continuamente pulsos de 25 fs (FWHM), centrados em 775 $\mathrm{nm}$ com $40 \mathrm{~nm}$ de largura de banda, taxa de repetição máxima de $4 \mathrm{kHz}$ e energia máxima por pulso de $750 \mu \mathrm{J}$.

Após a geração do feixe laser no laboratório de lasers de altíssima intensidade, ele é direcionado para o laboratório de aplicações industriais, percorrendo 
aproximadamente $30 \mathrm{~m}$ até um sistema de guiamento composto por obturadores, expansores, polarizadores, espelhos e todo um sistema óptico que permite o controle da dispersão a da intensidade do feixe, que é focalizado sobre a amostra por uma lente com comprimento focal de $40 \mathrm{~mm}$, gerando um diâmetro no foco (cintura) de aproximadamente $15 \mu \mathrm{m}$.

\subsection{Sistema de movimentação da amostra}

Para realizar a varredura da amostra com o feixe de laser focalizado, a estação de trabalho conta com uma base para fixação de amostras, que possui um sistema de movimentação tridimensional (eixos $x, y$ e $z$ ), com precisão submicrométrica controlado por computador, o que permite a execução de microusinagens com perfis complexos, por meio de programas de usinagem gerados por softwares específicos.

\subsection{Sistema de controle do espectro característico emitido pelo plasma durante a usinagem}

O controle da camada do material ablacionado foi realizado através da técnica de espectrometria de emissão atômica induzida por laser (LIBS - Laser-induced Breakdown Spectroscopy), que consiste na medição dos comprimentos de onda emitidos pelo plasma, classificando o material de origem a partir de seus picos característicos.

A coleta do sinal do plasma foi realizada por uma lente com diâmetro de $25 \mathrm{~mm}$, e distância focal de $50 \mathrm{~mm}$, a qual estava acoplada um cabo de fibra ótica para direcionar a luz captada até um espectrômetro modelo CCS100 fabricado pela Thorlabs, com uma faixa de medição de 350 à $700 \mathrm{~nm}$ de comprimento de onda, que gera os espectros num software próprio.

\subsection{Microscopia eletrônica de varredura (MEV)}

Todas as usinagens realizadas foram inicialmente analisadas por microscopia eletrônica de varredura, para avaliação do aspecto do fundo dos rebaixos ablacionados, bem como das bordas, para identificação de material ressolidificado ou ejetado da região da ablação.

O equipamento utilizado foi um microscópio de bancada modelo TM3000 fabricado pela Hitachi, com acessórios para análise de imagem em 2D e 3D, além de detectores para realização de Espectrometria de Energia Dispersiva (EDS), para identificação química.

\subsection{Espectrometria de fluorescência de raios-X (XRF)}

Para identificação das espécies químicas presentes nas amostras, tanto para avaliação e planejamento dos processos, como para avaliação de alguns resultados obtidos pelo LIBS, foi utilizado um espectrômetro de fluorescência de raios-X por energia dispersiva EDX-720 fabricado pela Shimadzu, onde foi possível quantizar os elementos presentes nas amostras antes e depois das ablações. 


\section{RESULTADOS E DISCUSSÃO}

\subsection{Posicionamento do sistema de controle LIBS}

Devido a baixa intensidade esperada do plasma gerado na ablação, foi necessário analisar o melhor posicionamento para o sistema LIBS. Utilizou-se um chapa de titânio GR 2, medindo $2 \times 10 \times 15 \mathrm{~mm}$ com a intenção de testar o posicionamento das lentes de captação do sinal do plasma e, simultaneamente, calibrar o espectrômetro para o material de interesse. A Figura 2 mostra os canais ablacionados na chapa de titânio, e traz uma representação do posicionamento das lentes para o teste de captação de sinal do plasma.

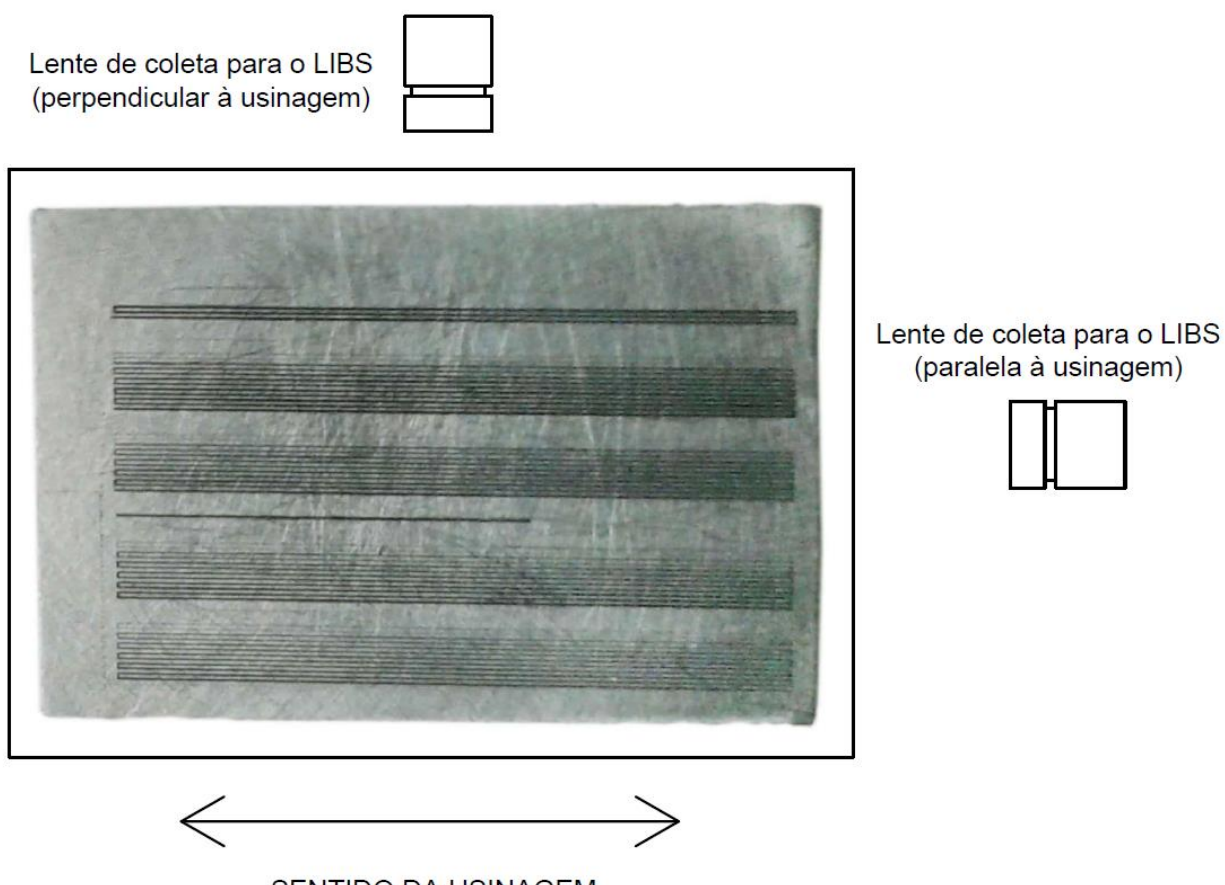

Figura 2. Posicionamento das lentes para teste de captação do sinal do plasma para o sistema LIBS.

Foram ablacionados quatro conjuntos de canais, variando a velocidade de avanço da usinagem de um para outro, e também, reduzindo a energia do laser de um canal para outro. Para verificar o melhor posicionamento os testes foram realizados posicionando as lentes para captação do sinal do plasma à frente e atrás do feixe laser, paralelas ao sentido da usinagem, e também perpendiculares ao movimento de varredura.

A Figura 3 mostra a comparação entre as intensidades relativas do sinal coletado para um pico característico do titânio, centrado num comprimento de onda aproximado de $453 \mathrm{~nm}$, mantendo-se todos os parâmetros de usinagem, exceto o posicionamento das lentes de coleta do sinal do plasma. 


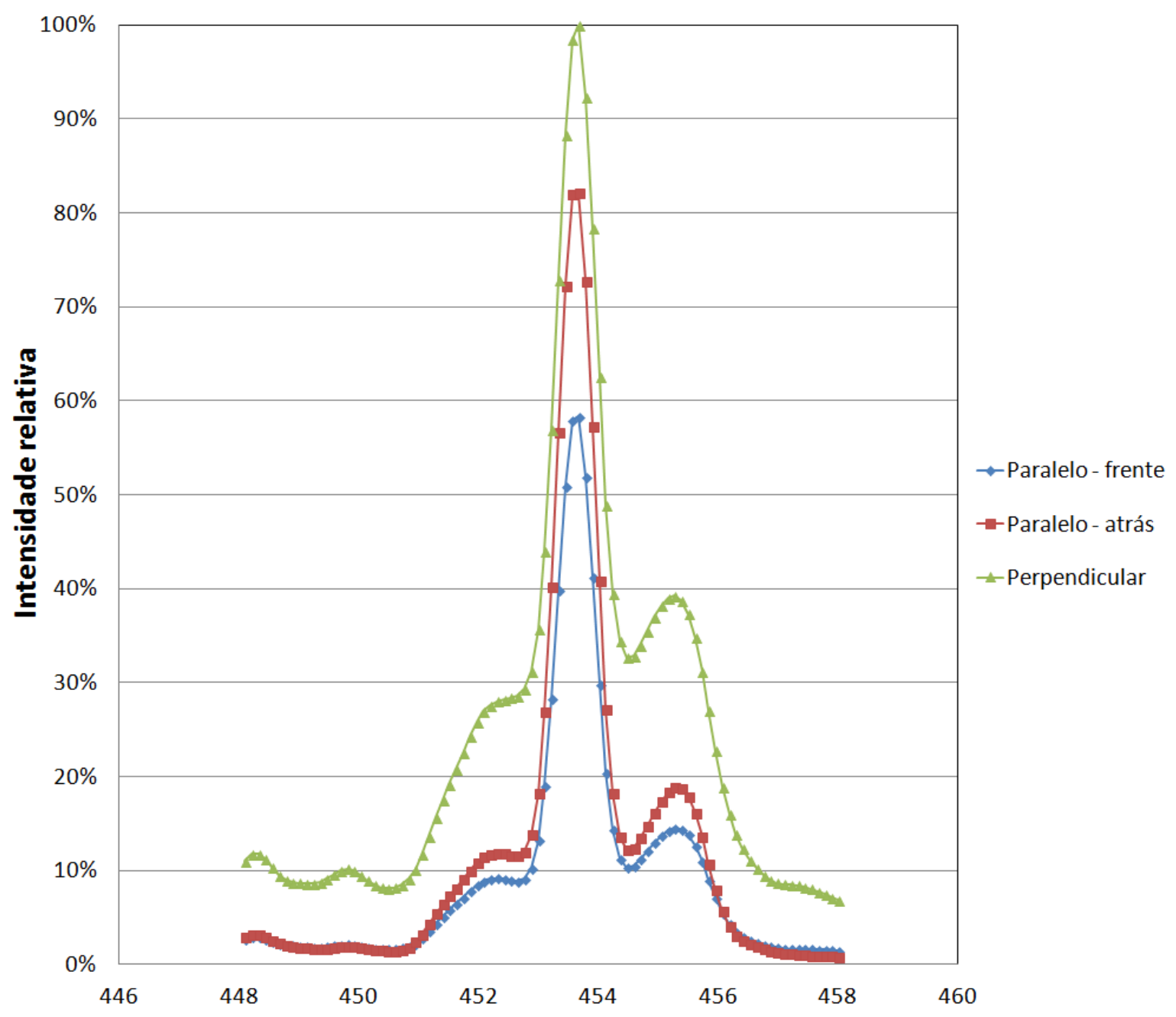

Comprimento de onda

Figura 3. Gráfico da intensidade relativa do sinal do plasma captada para cada configuração de posicionamento testada.

Após a análise, optou-se pela por posicionar o sistema de captação paralelo ao sentido da usinagem atrás do feixe, pois, apesar de apresentar intensidade menor que a montagem perpendicular, permitiu grande redução do sinal de background, o que permitiria fácil visualização do surgimento de novos picos, referentes ao material de base, no decorrer da ablação.

\subsection{Teste do sistema LIBS numa pastilha de usinagem}

Antes de realizar o procedimento para determinação do limiar de ablação do TiN e do WC, o sistema de controle LIBS foi testado utilizando-se uma pastilha de usinagem com recobrimento, porém sem especificação do recobrimento nem do material de base. Foram ablacionados cinco rebaixos de $2 \times 4 \mathrm{~mm}$, sendo que 0 primeiro foi feito em apenas um passe, o segundo em dois passes, e assim por diante. Os parâmetros das usinagens foram definidos de acordo com os testes realizados anteriormente na chapa de titânio, a energia do laser foi ajustada para $9,75 \mu \mathrm{J}$ por pulso e a velocidade de avanço foi de $100 \mathrm{~mm} / \mathrm{min}$. O gráfico da Figura 4 mostra a evolução da intensidade medida pelo LIBS, referente ao pico característico do titânio, durante a ablação da pastilha de usinagem. 


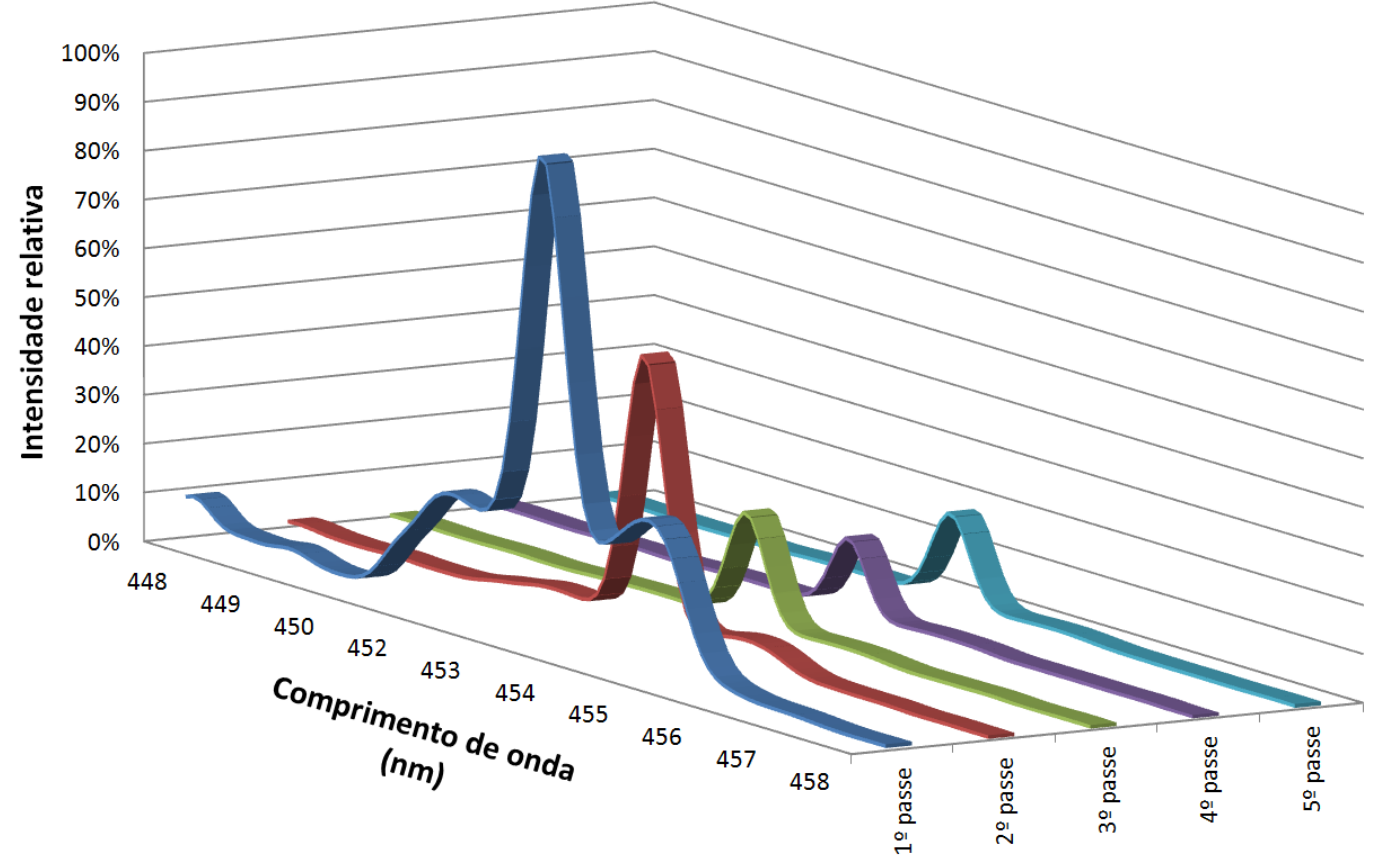

Figura 4. Evolução da intensidade do pico referente ao titânio.

Apesar da perceptível redução do sinal do titânio do primeiro para o último passe, percebe-se que a intensidade estabilizou-se por volta de 15\% da inicial, mesmo a profundidade do rebaixo tendo ultrapassado completamente o filme de recobrimento. Concluiu-se que a pastilha utilizada devia conter certa quantidade de titânio na composição de seu material de base, fato que foi confirmado após uma análise química do material por fluorescência de raios-X. A Tabela 1 apresenta os materiais presentes bem como suas concentrações aproximadas.

Tabela 1. Composição do substrato da pastilha utilizada para teste do sistema LIBS

\begin{tabular}{lllll}
\hline Elemento & $\begin{array}{c}\text { Concentração } \\
\text { relativa }(\%)\end{array}$ & Erro Linha intensidade
\end{tabular}

\begin{tabular}{lcccc}
\hline $\mathrm{Ti}$ & 60,739 & 0,146 & $\mathrm{TiKa}$ & 8,6467 \\
\hline $\mathrm{W}$ & 32,521 & 0,087 & $\mathrm{WLa}$ & 6,8959 \\
\hline $\mathrm{Co}$ & 5,094 & 0,026 & $\mathrm{CoKa}$ & 1,8621 \\
\hline $\mathrm{Nb}$ & 1,646 & 0,010 & $\mathrm{NbKa}$ & 1,3165
\end{tabular}

A partir desses resultados, concluiu-se que o sistema de controle LIBS que foi montado é capaz de identificar o material ablacionado, bem como evidenciar a redução da concentração de um material e o aumento de outro, porém, limitados pela sensibilidade do equipamento, será difícil identificar com precisão a transição do material do filme para o material do substrato, tendo em vista que o plasma gerado apresenta intensidade baixa, devido às baixas intensidades do laser.

\subsection{Determinação do limiar de ablação do TiN}

Para determinação do limiar de ablação do TiN foi utilizada uma pastilha de usinagem de metal duro recoberta com um filme de aproximadamente $5 \mu \mathrm{m}$ de 
espessura, adquirida diretamente com o fornecedor, que não informou a composição exata do substrato e nem do filme.

O procedimento do D-Scan foi realizado no Laboratório de Lasers de Altíssima Intensidade do CLA-IPEN, onde foram feitos 24 traços na superfície da amostra, com a energia do laser variando de 97 à $91 \mu \mathrm{J}$. A Figura 5 mostra os perfis gravados na superfície da pastilha.

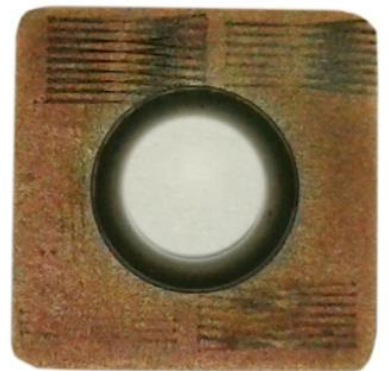

Figura 5. Perfis gravados na pastilha de usinagem pelo método D-Scan para determinação do limiar de ablação do filme de TiN.

As imagens de cada perfil foram ampliadas utilizando um MEV e, posteriormente, as dimensões máximas e mínimas da espessura de cada perfil foram registradas para determinação do limiar de ablação utilizando as equações definidas no D-Scan.

Analisando o gráfico da Figura 6, pode-se verificar que o limiar de ablação, para o filme de TiN, gira em torno de $F_{t h}=0,9 \pm 0,14 \mathrm{~J} / \mathrm{cm}^{2}$, com um nível baixo de confiança, visto a grande dispersão de alguns valores, que estão em revisão.

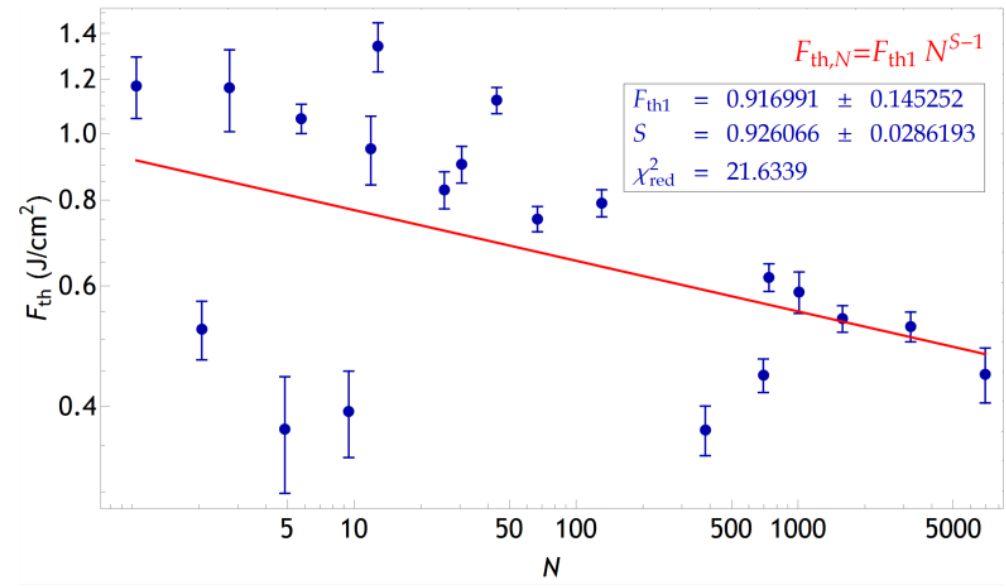

Figura 6. Gráfico do limiar de ablação para o filme de TiN no regime de baixa fluência, pelo método de D-Scan.

\subsection{Determinação do limiar de ablação do WC}

Para determinação do limiar de ablação do WC utilizou-se o mesmo tipo de pastilha adquirida para determinação do limiar do filme de TiN, uma de suas faces foi lixada, até lixa 600, para remoção do filme superficial, deixando assim exposto o material do substrato.

O procedimento do D-Scan foi realizado no Laboratório de Lasers de Altíssima Intensidade do CLA-IPEN, onde foram feitos 16 traços na superfície da amostra, com a energia do laser variando de 94 à $87 \mu \mathrm{J}$. A Figura 7 mostra o detalhe de um dos perfis gravados na superfície da pastilha. 


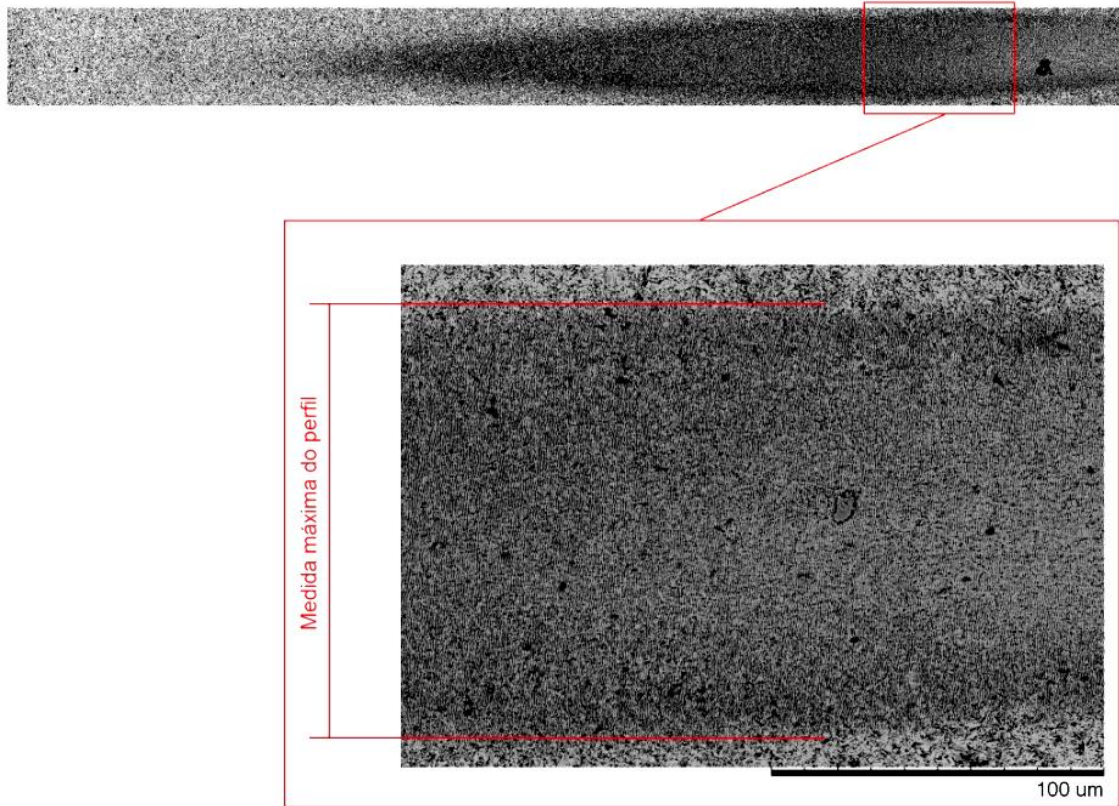

Figura 7. Perfil gravado na superfície da pastilha para determinação do limiar de ablação pelo método D-Scan. No detalhe, região de maior medida do perfil.

As imagens de cada perfil foram ampliadas utilizando um MEV e posteriormente as dimensões máximas e mínimas da espessura de cada perfil foram registradas para determinação do limiar de ablação utilizando as equações definidas no D-Scan.

Analisando o gráfico da Figura 8, pode-se verificar que o limiar de ablação, para o substrato de WC, gira em torno de $F_{t h}=0,6 \pm 0,14 \mathrm{~J} / \mathrm{cm}^{2}$, com um nível baixo de confiança, visto a grande dispersão de alguns valores, que estão em revisão.

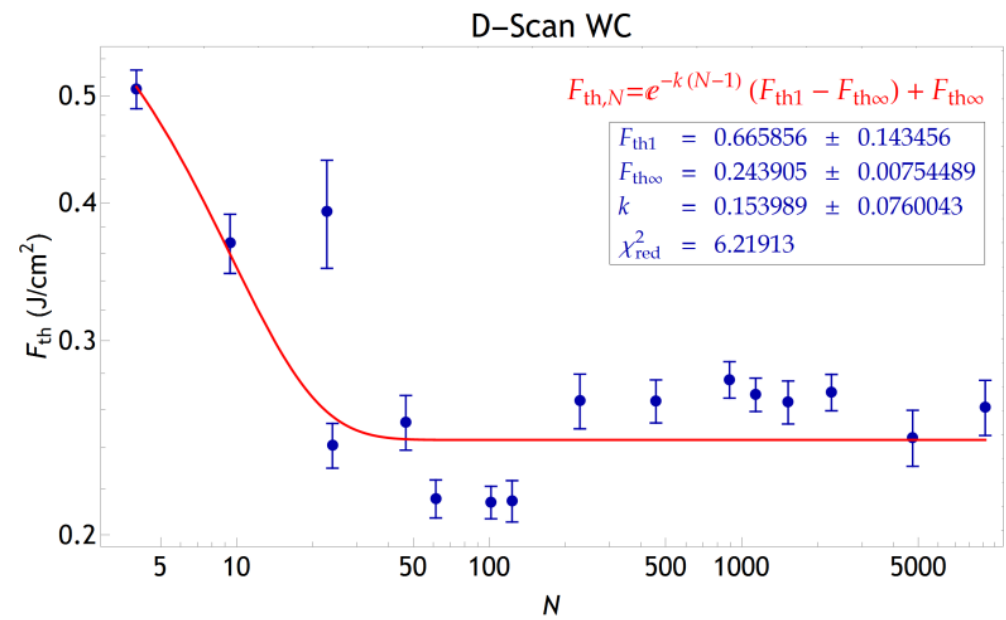

Figura 8. Gráfico do limiar de ablação para o filme de TiN no regime de baixa fluência, pelo método

\section{CONCLUSÃO} de D-Scan.

O sistema de controle LIBS montado para monitorar o material irradiado pelo laser no processo de ablação mostrou-se eficiente, capaz de identificar a redução gradativa de determinado material no decorrer da usinagem.

O limiar de ablação muito maior para o filme de TiN em relação ao do substrato de WC, determinados pelo método D-Scan, evidencia a dificuldade do processo, uma vez que, após eliminado o filme, o substrato seria irradiado pelo laser com intensidade muito acima da necessária para lhe causar danos. 
Estima-se que, após um fino ajuste do sistema de LIBS e a escolha ideal para os parâmetros do laser, seja possível remover gradativamente o filme de TiN da pastilha causando um mínimo de dano ao substrato de WC. Tais processos estão em execução, devendo ser finalizados e avaliados para então, discutir-se a possibilidade do processo.

\section{Agradecimentos}

Ao Instituto de Pesquisas Energéticas e Nucleares (IPEN) e ao Centro de Lasers e Aplicações (CLA), pela infra estrutura concedida para realização deste trabalho.

À todos os funcionários do CLA, em especial ao Prof. Dr. Wagner de Rossi, pela ajuda em todas as etapas do trabalho, e pela paciência e tranquilidade para indicar 0 caminho e entender as dificuldades.

\section{REFERÊNCIAS}

1 Ohring M. Materials science of thin films : deposition and structure. 2nd ed. San Diego, CA: Academic Press; 2002. xxi, 794 p. p.

2 Ohring M. The materials science of thin films. Boston: Academic Press; 1992. xx, 704 p. p.

3 Malinauskas M, Danilevičius P, Juodkazis S. Three-dimensional micro-/nano-structuring via direct write polymerization with picosecond laser pulses. Optics express. 2011;19(6):5602-10.

4 Miziolek AW, Palleschi V, Schechter I. Laser induced breakdown spectroscopy: Cambridge University Press; 2006

5 Xiao SZ, Schops B, Ostendorf A. Selective Ablation of Thin Films by Ultrashort Laser Pulses. Laser Assisted Net Shape Engineering 7 (Lane 2012). 2012;39:594-602.

6 Rublack T, Hartnauer S, Kappe P, Swiatkowski C, Seifert G. Selective ablation of thin $\mathrm{SiO} 2$ layers on silicon substrates by femto- and picosecond laser pulses. Materials Science \& Processing. 2011;103(1):43-50.

7 Samad RE, Vieira Jr N. Geometrical method for determining the surface damage threshold for femtosecond laser pulses. Laser physics. 2006;16(2):336-9.

8 Machado LM, Samad RE, de Rossi W, Vieira ND. D-Scan measurement of ablation threshold incubation effects for ultrashort laser pulses. Optics Express. 2012;20(4):4114-23. 